\title{
Lower Renal Threshold for Glucose Reabsorption in Type 1 Diabetes Mellitus (T1DM) May Explain the Smaller Contribution of SGLT2 Inhibitors to the Improvement of Plasma Glucose Control Compared with T2DM
}

\author{
Aya Osaki · Yoko Shimoda · Junichi Okada • Eijiro Yamada • \\ Tsugumichi Saito · Yasuyo Nakajima · Atsushi Ozawa • \\ Yawara Niijima · Shuichi Okada (D) - Masanobu Yamada
}

Received: April 25, 2019 / Published online: June 13, 2019

(c) The Author(s) 2019

\section{ABSTRACT}

Introduction: Previously, we reported that the renal threshold for glucose reabsorption can be measured as the lowest plasma glucose level that correlates with the first detectable appearance of urine glucose. These data revealed significant variations among patients with type 2 diabetes mellitus (T2DM), and there was a significant negative correlation between the renal threshold for glucose reabsorption and HbA1c levels following treatment with the sodiumglucose co-transporter 2 (SGLT2) inhibitor ipragliflozin. Recently approved SGLT inhibitors may not show the same efficacy in patients with T1DM as in those with T2DM unless the

Enhanced Digital Features To view enhanced digital features for this article go to https://doi.org/10.6084/ m9.figshare.8235704.

A. Osaki · Y. Shimoda $\cdot$ J. Okada $\cdot$ E. Yamada .

T. Saito · Y. Nakajima · A. Ozawa · Y. Niijima .

S. Okada $(\bowtie) \cdot$ M. Yamada

Department of Medicine and Molecular Science, Gunma University Graduate School of Medicine, 3-39-15 Showa-Machi, Maebashi, Gunma, Japan e-mail: okadash@gunma-u.ac.jp

J. Okada $\cdot$ Y. Niijima $\cdot$ S. Okada

Kan-Etsu Chuo Hospital, 71 Kitahara, Takasaki, Gunma, Japan renal threshold for glucose reabsorption shows similar levels between the two groups. SGLT2 inhibitors improve plasma glucose control in patients with T2DM by reducing glucose reabsorption via the epithelial cells of the proximal tubule.

Methods: The renal threshold for glucose reabsorption was defined as the minimum blood glucose concentration that results in the presence of measurable glycosuria in at least 12 measurements.

Results: The renal threshold for glucose reabsorption in patients with T2DM $[n=64$; $201.8 \pm 33.6$ (range 121-268) $\mathrm{mg} / \mathrm{dL}$ ] was significantly higher than that in patients with T1DM $[n=33 ; 171.0 \pm 33.0$ (range 76-259) $\mathrm{mg} /$ $\mathrm{dL} ; p=0.00022]$.

Conclusion: The renal threshold for glucose reabsorption in patients with T1DM was near the normal range and significantly lower than that in patients with T2DM. The efficacy of the SGLT2 inhibitor was better in patients with a higher renal threshold for glucose reabsorption. Thus, these results indicate that it is advisable to estimate the renal threshold for glucose reabsorption prior to initiating SGLT2 inhibitor therapy in patients with T1DM.

Keywords: Renal threshold for glucose reabsorption; Sodium-glucose co-transporter 2 inhibitor; Type 1 diabetes mellitus; Type 2 diabetes mellitus 


\section{INTRODUCTION}

Sodium-glucose co-transporter 2 (SGLT2) inhibitors improve plasma glucose control by reducing glucose reabsorption via the epithelial cells of the proximal tubule in patients with type 2 diabetes mellitus (T2DM) [1, 2]. Thus, SGLT2 inhibitors reduce HbA1c levels by decreasing the renal threshold for glucose reabsorption in these patients. Because the renal threshold of $200-250 \mathrm{mg} / \mathrm{dL}$ in these patients is higher than that in normal subjects (170-200 mg/dL), SGLT2 inhibitors are effective and promising oral glucose-lowering drugs $[1,2]$. However, in case of treatment with the SGLT2 inhibitor ipragliflozin, there is a significant negative correlation between the renal threshold for glucose reabsorption and HbA1c levels [3]. Recently, two SGLT2 inhibitors received approval for the treatment of patients with T1DM: dapagliflozin in Europe and ipragliflozin in Japan. Despite their availability, a comparative analysis of the effectiveness of SGLT2 inhibitors between T1DM and T2DM is lacking. Therefore, we compared the renal threshold for glucose reabsorption between patients with T1DM and those with T2DM.

\section{METHODS}

\section{Participants}

The study protocol was reviewed and approved by the review board of Gunma University in accordance with the principles of the Declaration of Helsinki. Written informed consent was obtained to analyze and report the patients' clinical laboratory data. The study included 64 and 33 patients with T2DM and T1DM, respectively.

\section{Study Design}

Prior to their hospital visit, patients were asked to empty their bladders as much as possible, and urine samples were collected just before drawing patient blood samples. Follow-up examinations were performed at the hospital once a month, and the blood samples were obtained to measure blood glucose and HbA1c levels. In addition, urinary glucose levels were determined in spot urine samples at each visit, as previously described [3]. Patients were monitored for 12 months. Venous blood samples were collected into tubes containing ethylenediaminetetraacetic acid and fluoride. Plasma glucose and HbA1c levels were determined from the plasma separated from the whole blood within an hour after collection, according to the hexokinase method using a Synchro CX4/CX5 glucose analyzer (Beckman Coulter Inc. Fullerton, CA, USA) and the Glycohemoglobin Analyzer RC20 (Sekisui Medical Co., Ltd, Tokyo, Japan) respectively. The intra- and inter-assay coefficients of variation were at most $2 \%$ at plasma glucose values of less than $126 \mathrm{mg} / \mathrm{dL}$ [3].

The renal threshold for glucose reabsorption was defined as the minimum plasma glucose concentration that resulted in the presence of measurable urine in at least 12 measurements. Blood glucose levels were placed in descending order of power aligned in the left to right direction with urinary glucose concentration results. The renal threshold for glucose reabsorption was determined as the minimum blood glucose level at the turning point from the absence to presence of urinary glucose [3].

\section{Statistical Analysis}

Results are expressed as mean \pm standard deviation. All statistical analyses were performed using the InStat 2 program.

\section{RESULTS}

\section{Patient Characteristics}

The median age of the subjects was 64 (range 46-83) and 36 (range 24-75) years for patients with T2DM and T1DM, respectively. The median systolic blood pressure was 131.0 (range 109-156) and 118.0 (range 102-136) $\mathrm{mmHg}$, median diastolic blood pressure was 72.5 (range 60-90) and 75.0 (range 64-88) $\mathrm{mmHg}$, and 
median body weight was 66.65 (range $54.0-98.5$ ) and 60.6 (range $44.0-72.5) \mathrm{kg}$ in patients with T2DM and T1DM, respectively. The median duration of T2DM and T1DM diabetes was 5.6 (range 3-18) and 16.4 (range 1-33) years, respectively.

\section{Evaluation of Renal Threshold for Glucose Reabsorption}

The renal threshold for glucose reabsorption in patients with T2DM $[201.8 \pm 33.6$ (range $121-268) \mathrm{mg} / \mathrm{dL}$ ] was significantly higher than in those with T1DM [171.0 \pm 33.0 (range 76-259) $\mathrm{mg} / \mathrm{dL} ; p=0.00022$; Fig. 1].

\section{DISCUSSION}

We previously reported that in patients with T2DM, there is a significant negative correlation between the renal threshold for glucose reabsorption and HbA1c levels following treatment

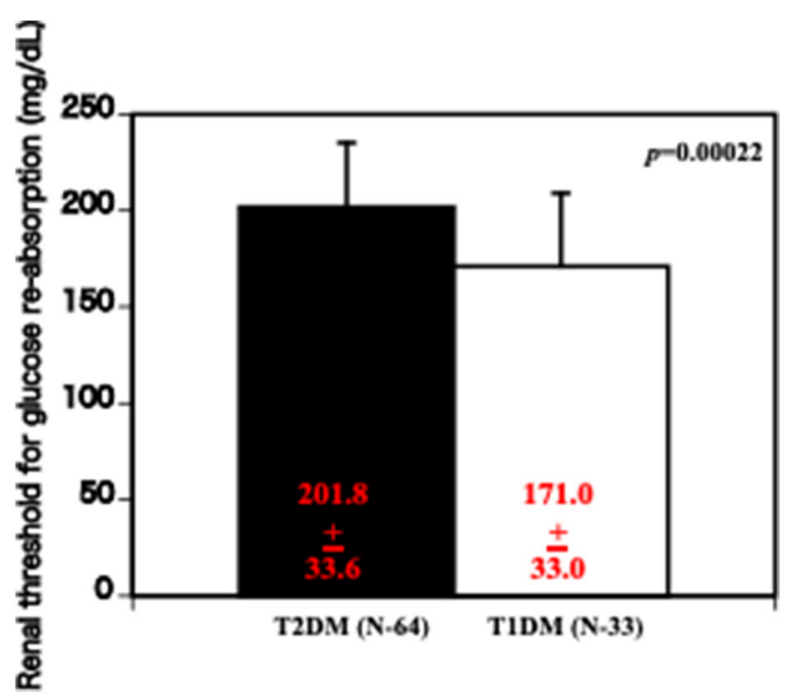

Fig. 1 Comparison of the renal threshold for glucose reabsorption between patients with T1DM and T2DM. The $y$-axis represents the renal threshold for glucose reabsorption $(\mathrm{mg} / \mathrm{dL})$. Patients with T1DM show a significantly lower renal threshold for glucose reabsorption than patients with T2DM $(201.8 \pm 33.6$ vs. $171.0 \pm 33.0, p=0.00022)$. Closed bars and open columns represent the threshold in patients with T2DM and T1DM, respectively with the SGLT2 inhibitor ipragliflozin [3], i.e., the efficacy of the SGLT2 inhibitor is better in patients with a higher renal threshold for glucose reabsorption. Because the capacity to reabsorb urinary glucose via the epithelial cells of the proximal tubule is poor in patients with lower renal threshold for glucose reabsorption, these patients regularly excrete urinary glucose even at lower ranges of plasma glucose. Conversely, patients with a higher renal threshold for glucose reabsorption have a greater capacity to reabsorb urinary glucose, and they tend to excrete urinary glucose only at higher ranges of plasma glucose. Unexpectedly, our findings indicated that the renal threshold for glucose reabsorption in patients with T1DM was near the normal range and significantly lower than that in patients with T2DM. This suggests that SGLT2 inhibitors might not be as effective in treating patients with T1DM as in those with T2DM. The improvement in HbA1c level after initiating SGLT2 inhibitor treatment, expressed as a decrease from baseline, was $0.42 \%$ and $0.82 \%$ in patients with T1DM and T2DM, respectively $[4,5]$. Thus, these results suggest that SGLT2 inhibitors effectuated plasma glucose control in patients with T1DM to a lesser degree than in those with T2DM. Thus, physicians are advised not to have high expectations regarding the efficacy of SGLT2 inhibitors in patients with T1DM.

There are several limitations of the study. First, the sample size was small, affecting the generalizability of the results; therefore, these results should be confirmed in a larger number of subjects. Second, the study has a selection bias because of the ethnicity of the participants. Third, the difference in the duration of the disease between patients with T1DM and in those with T2DM may have generated a bias that was not accounted for.

\section{CONCLUSION}

A significant negative correlation between the renal threshold for glucose reabsorption and HbA1c levels following treatment with the SGLT2 inhibitor ipragliflozin was established. We report that the renal threshold for glucose 
reabsorption in patients with T1DM is near the normal range and significantly lower than that in patients with T2DM. Thus, it is advisable to estimate the renal threshold for glucose reabsorption prior to initiating SGLT2 inhibitor therapy in patients with T1DM.

\section{ACKNOWLEDGEMENTS}

The authors thank all participants of the study.

Funding. No funding or sponsorship was received for this study or publication of this article. The article processing charges were funded by the authors.

Editorial Assistance. The authors would like to thank MARUZEN-YUSHODO Co., Ltd. (http://kw.maruzen.co.jp/kousei-honyaku/) for the English language editing. English language editing was funded by the authors.

Authorship. All named authors meet the International Committee of Medical Journal Editors (ICMJE) criteria for authorship for this article, take responsibility for the integrity of the work as a whole, and have given their approval for this version to be published.

Disclosures. Aya Osaki, Yoko Shimoda, Junichi Okada, Eijiro Yamada, Tsugumichi Saito, Yasuyo Nakajima, Atsushi Ozawa, Yawara Niijima, Shuichi Okada, and Masanobu Yamada have nothing to disclose.

Compliance with Ethics Guidelines. The study protocol was reviewed and approved by the review board of Gunma University in accordance with the principles of the Declaration of Helsinki. Written informed consent was obtained to analyze and report the patients' clinical laboratory data.
Data Availability. The datasets generated during and/or analyzed during the current study are available from the corresponding author on reasonable request.

Open Access. This article is distributed under the terms of the Creative Commons Attribution-NonCommercial 4.0 International License (http://creativecommons.org/licenses/ by-nc/4.0/), which permits any noncommercial use, distribution, and reproduction in any medium, provided you give appropriate credit to the original author(s) and the source, provide a link to the Creative Commons license, and indicate if changes were made.

\section{REFERENCES}

1. Lupsa BC, Inzucchi SE. Use of SGLT2 inhibitors in type 2 diabetes: weighing the risks and benefits. Diabetologia. 2018;61:2118-25.

2. DeFronzo RA, Hompesch M, Kasichayanula SK. Characterization of renal glucose reabsorption in response to dapagliflozin in healthy subjects and subjects with type 2 diabetes. Diabetes Care. 2013;36:3169-76.

3. Osaki A, Okada S, Saito T. Renal threshold for glucose reabsorption predicts diabetes improvement by sodium-glucose cotransporter 2 inhibitor therapy. J Diabetes Investig. 2016;7:751-4.

4. Strojek K, Yoon KH, Hruba V. Effect of dapagliflozin in patients with type 2 diabetes who have inadequate glycaemic control with glimepiride: a randomized, 24-week, double-blind, placebo-controlled trial. Diabetes Obes Metab. 2011;13:928-38.

5. Mathieu C, Dandona P, Gillard P. Efficacy and safety of dapagliflozin in patients with inadequately controlled type 1 diabetes (the DEPICT-2 Study): 24-week results from a randomized controlled trial. Diabetes Care. 2018;41:1938-46. 